\title{
Explanatory models in psychiatry
}

\section{Citation}

Weiss, Mitchell G and Daryl Somma. 2007. Explanatory Models in Psychiatry. In Textbook of Cultural Psychiatry, eds. Dinesh Bhugra and Kamaldeep Bhui, pp. Cambridge; Cambridge University Press.

\section{Published Version}

doi:10.1017/cbo9780511543609.012

\section{Permanent link}

http://nrs.harvard.edu/urn-3:HUL.InstRepos:23593201

\section{Terms of Use}

This article was downloaded from Harvard University's DASH repository, and is made available under the terms and conditions applicable to Other Posted Material, as set forth at http:// nrs.harvard.edu/urn-3:HUL.InstRepos:dash.current.terms-of-use\#LAA

\section{Share Your Story}

The Harvard community has made this article openly available.

Please share how this access benefits you. Submit a story.

\section{Accessibility}




\title{
Explanatory models in psychiatry
}

\author{
Mitchell G. Weiss and Daryl Somma
}

\section{EDITORS' INTRODUCIION}

Similar experiences of illness or distress are seen as being caused by different elements across different cultures. Feeling gutted and sinking heart are idioms of distress which are remarkably different across cultures, but their implications at an individual level are very similar. Furthermore, the causation of the distress will be seen as remarkably different. It is evident that, in traditional cultures, the locus of control may be seen as external which may be coloured by cultural expectations, whereas in others the locus of control may be internal. Within each culture, how. ever, individuals may carry their own explanations which may or may not be strongly influenced by individual's culture. From a clinical perspective, it is crucial that clinicians are aware of explanatory models that patients bring to the therapeutic encounter so that engagement can begin.

Weiss and Somma examine the concepts of the explanatory model framework, its appeal to health professionals and social scientists as well as its limitations. The illness explanatory framework deals with notions about an episode of illness and its treatment by all who are engaged in the clinical process and understanding these models means that patients' views on their conditions are being acknowledged. Weiss and Somma emphasize that the model must be distinguished from other ways the term is used which may refer to the nature of health and other problems in general. They explain three formulations of illness explanatory models and describe conceptual underpinning of the illness explanatory framework. In the beginning of the illness explanatory model framework provided a means of bring cultural differences between patients and clinician (especially when they came from different ethnic and cultural backgrounds) in multicultural settings. However, Weiss and Somma illustrate that this approach should also be seen as managing concerns about an imbalance over-emphasizing biology in the biopsychosocial model. They are also aware of possible critique of the model. This critique includes the perceived fixed and static nature of the model and that clinical interests do not take into account the influence of social context. Perhaps the most significant criterion is based on the question of whether too much emphasis on the model in explanatory model exists. Models are important for developing theory and for research. The approach remains a useful one for bridging the interests and experiences of clinicians and their patients.

\section{Introduction}

Over the past three decades the illness explanatory model framework has stimulated research in clinically applied medical anthropology, guided clinical training, sparked controversy in the health social sciences and guided developments in the field of cultural psychiatry. This formulation of explanatory models was conceived both to advance perspectivism in clinical medical practice and public health, and to show how ethnomedical study of sickness and medicine should contribute to cultural anthropology and social analysis. The appeal of the explanatory model framework for clinical training is based on the premise that it is important to examine relationships and consequences of interactions between patients' ideas about their health problems and those of clinicians and professionals who are responsible for their care. Although the clinical interests and applications of illness explanatory models extend to all aspects of medicine, it was mainly experience and interest in psychiatry and 
culture, and their effects on medical practice through consultation liaison, that spurred initial interest and development of the illness explanatory model framework. In bridging the interests of psychiatry, medicine and medical anthropology its influence has been unrivalled.

This chapter examines the concept and underpinnings of the explanatory model framework, its appeal to health professionals and social scientists and its limitations. Because it was defined in very simple terms and applied to so many clinical and social science questions, the idea of illness explanatory models means different things to different people, and various ambiguities and misinterpretations have resulted. Consequently, some psychiatrists, other health professionals and social scientists have been wary of its influence and the possibility of overselling its significance, and we examine the nature of their critiques. The chapter concludes with a discussion of the current role of explanatory models in cultural psychiatry, approaches to studying explanatory models and their influence on the development of cultural epidemiology.

\section{What are illness explanatory models?}

The illness explanatory model framework as it is now commonly understood developed in the late 1970 s, and it is associated most closely with the influence of Arthur Kleinman and networks in cultural psychiatry, medicine and medical anthropology. Among various accounts in the literature, one that is frequently cited defines the illness explanatory model as 'notions about an episode of sickness and its treatment that are employed by all those engaged in the clinical process', (Kleinman, 1980, p. 105). Interest in explanatory models was equally concerned with everyone involved in clinical encounters. At the outset, inasmuch as explanatory models were expected to provide a framework to guide ethnomedical study of societies and health systems, attending to 'the conceptions of sickness held by patients, communities, practitioners, and researchers' was essential (Kleinman, 1977a). As a clinical interest, explanatory models were particularly notable because they acknowledged the significance of patients' points of view as complementary to health professionals' assessments. In that sense, they referred not so much to formal structural or predictive models, but rather to the way people think and speak, as a way of explaining illness (Helman, 2004).

This formulation must be distinguished from other ways the term explanatory model is used, which may refer to the nature of health (and other) problems in general, rather than anchored to specific illness episodes and experiences. Social scientists and empirical researchers in many fields commonly apply the term explanatory model to statistical models, analysis of epidemiological patterns and theoretical propositions about a wide range of phenomena. If we distinguish studies of Kleinman's illness explanatory models from these generic non-illness explanatory model studies, one finds the latter are far more frequent in the medical literature (e.g. 'Crowding and violence on psychiatric wards: explanatory models'; 'A test of two explanatory models of women's responses to battering'; 'Explanatory model to describe school district prevalence rates for mental retardation and learning disabilities'). When we examined 677 Medline references with the term explanatory model or explanatory models in their title or abstracts in March 2006, we found 181 of these citations were for articles concerned with illness explanatory models, and 62 were concerned with mental-health problems (Table 10.1).

The illness explanatory models of patients, family, doctors and others concerned with health problems and the clinical process did not refer to a professionally elaborated explanatory theory or to research findings derived from empirical study of explanatory variables and outcome measures, even though they might be influenced by them. Instead, these explanatory models were representations of illness, described with reference to a set of cognitive explanations; symptomatic, emotional and social experiences; and to prototypic personal history and associations that collectively characterized the 
Table 10.1. Medline illness explanatory model studies and their focus on psychiatric problems over successive 5-year periods

\begin{tabular}{lcccc}
\hline Period & \multicolumn{2}{c}{ All illness EMs } & & \multicolumn{2}{l}{ Mental-health problem EMs } \\
\cline { 5 - 5 } \cline { 5 - 5 } & $N$ & & $N$ & Percentage \\
\hline $1976-1980$ & 1 & & 0 \\
$1981-1985$ & 14 & 1 & 7.1 \\
$1986-1990$ & 25 & 7 & 28.0 \\
$1991-1995$ & 30 & & 12 & 40.0 \\
$1996-2000$ & 50 & & 19 & 38.0 \\
$2001-2005$ & 61 & 23 & 37.7 \\
Total & 181 & 62 & 34.3 \\
\hline
\end{tabular}

illness at a particular point of inquiry. Clinical writing described explanatory models variously as ideas, notions, or beliefs about 'the nature, name, cause, expected course, and desired treatment for an episode' of illness (Kleinman, 1986, p. 84). These notions were responsive to fundamental questions concerning the why, what, and how of illness: 'Why me? Why now? What's wrong? How long will it last? How serious?' (Kleinman, 1988b, p. 156).

\section{Three formulations of illness explanatory model}

Efforts to make social-science concepts accessible in a clinically applied medical anthropology, as well as a process of ongoing rethinking of the role of explanatory models in health social science research beyond their clinical interests, have also led to ambiguities. Consequently, the significance and even the nature of explanatory models are understood differently by various authors. One may discern three relatively distinct formulations, each with some interest in both representational and predictive features of explanatory models. In its narrowest sense, the illness explanatory model is concerned primarily or exclusively with an account of the reasons for illness, that is, causal attributions or perceived causes. In that sense, it is less concerned with experience, behaviour or social factors as contexts, unless they are identified as causes. This conceptually scaled-down version of explanatory models may simplify the clinical ethnographic enterprise to a degree that seems simplistic from an anthropological perspective. On the other hand, for clinicians with little interest in engaging in social science research, or even in any kind of research, working with a clear operational definition enhances the appeal of this formulation.

Another view of explanatory models is more comprehensive and aims to be truer to the ethnographic interests that initially motivated interest in a task in clinical ethnography. This broad formulation eschews the idea of an explanatory model concerned solely with perceived causes; they may be an important part, but they are not the whole of an illness explanatory model. Referring to important contributions of Byron Good (1977) to the early development of the concept, Kleinman wrote:

'Patient and family EMs often do not possess single referents but represent semantic networks that loosely link a variety of concepts and experiences' (Kleinman, 1980, pp. 106-107, see also p. 108, fig. 4).

The role of a semantic network analysis, rooted in ideas of causal webs and the influence of social networks, was complementary to the interests in cognitive accounts of perceived causes. In the early phase of its development, illness explanatory models were expected to link the clinical process to ethnographic interests, a means of constructing new models for medical anthropology (Kleinman, $1978 \mathrm{~b}$ ), and advancing the interests of ethnomedicine (Fabrega, 1975).

Inasmuch as features of the clinical applications and the nature of the inquiry somewhat resembled the process of exploratory psychotherapy, psychiatrists were especially interested and influential in developing the approach. The explanatory model framework, however, differed markedly from standard psychiatric practice on several counts. In response to the tension between accepting a fixed nosological standard and acknowledging the influence of culture and context, standard psychiatric 
paradigms were no longer merely authoritative tools for clinical assessment. They also became the objects of study and a process of rethinking that was concerned with the validity and utility of interpretive models of psychoanalysis, phenomenological diagnostic assessment and the biology of mental disease.

The third formulation of the illness explanatory model was concerned with the intricacies and expectations from modelling the interaction of explanatory models of patients and others involved in the clinical process, especially their doctors or other healers. Although this model of the interacting patient and healer models was largely representational, it was also presented with a promise for empirical study that would test its validity based on whether it could predict the course of the clinical process. In that sense, it was comparable to the generic interests of other non-illness explanatory models. In his seminal exposition of the illness explanatory model framework, Kleinman proposed analysing clinical encounters with reference to the explanatory models of patients $\left(\mathrm{EM}_{\mathrm{p}}\right)$, their family $\left(\mathrm{EM}_{\mathrm{f}}\right)$, and their doctors $\left(\mathrm{EM}_{\mathrm{d}}\right)$, each of which might be multiple (EMp.1,p.2.., etc.) (Kleinman, 1980, p. 112, fig. 5). He advocated empirical studies to test hypotheses concerned with how these models interact, especially the premise that congruent models would result in better clinical outcomes, and that incongruent models require negotiation.

\section{Conceptual underpinnings}

The priority of the illness explanatory model framework, with its appreciation of the relevance of patients' and healers' points of view and various ways that cultural contexts influence both, was related to important parallel and prior developments. These included Leon Eisenberg's (1977) distinguishing fundamental features of disease and illness that highlighted limitations of exclusively biological and technical approaches to medical practice. The work of George Engel (1977) focused on advancing the biopsychosocial model of psychiatry and medicine, arguing that each perspective alone was inadequate, and together they were complementary. Although Eisenberg and Engel were each responding to identified problems in American medicine, their contributions were also applicable to a dilemma that resulted from a daunting rift between biological reductionist and cultural relativist approaches. Kleinman had identified these as an inescapable feature of anthropological and cross-cultural health studies (Kleinman, 1977a).

Kleinman also regarded the illness explanatory framework as one among a group of contributions to an 'ethnomedical programme'. Like Eisenberg's formulation of disease and illness, the semantic illness network model of Good (1977) was closely related to the interests, substance and tasks of the illness explanatory model framework. Other relevant work included Fabrega's enduring interest in ethnomedicine and ethnomedical models (Fabrega, 1990). These were complementary and shared interests, but each with a distinctly different focus and appeal: explanatory models for bringing anthropology to medicine, and ethnomedicine for bringing medicine to anthropology.

Another important influence was given less attention in the literature on explanatory models than may have been warranted. The ideas and contributions of the so-called emic-etic paradigm have now become so well accepted as a tool for social research that it is difficult to appreciate how controversial they were several decades ago. Kenneth Pike developed this framework acknowledging the insider's and the outsider's perspective for social analysis from experience studying previously unknown languages without a bilingual guide (Pike, 1967; Headland, Pike and Harris, 1990). Drawing on basic linguistic concepts, he developed a paradigm for cultural study and social analysis. From the concept of phoneme and phonemic analysis, referring to the basic unit of meaning within a language, valid with reference to a particular language, came the notion of emic. A phonetic analysis of language, on the other hand, examines basic units of sound with reference to phonological universals, as conceptualized by linguists independent of any particular 
language. In the disease/illness and explanatory model paradigms of Eisenberg and Kleinman, this perspectivist view was brought into health and medical studies, clinical practice and training. Although the focus on culture at large and on individual patients or individuals may differ, the task of eliciting explanatory models shares a common interest in elaborating an emic account of health problems.

\section{Developing the explanatory model framework}

The decade from the late 1970s was an important period in the development and promotion of the illness explanatory model framework. Much of the subsequent literature on the topic has referred to Kleinman's (1980) book, Patients and Healers in the Context of Culture, and its paradigmatic questions have guided considerable clinical training and research on the topic (p. 104n). The intellectual landscape, however, had already been diligently paved with a series of publications in key journals of their respective fields that indicated practical approaches for clinical medical anthropology in psychiatry (Kleinman, 1978a) and internal medicine. Theoretical contributions arguing for needed models in medical anthropology (Kleinman, 1978b) and for development of the 'new cross-cultural psychiatry' (Kleinman, 1977b) also referred to explanatory models, but sparingly. The term figured far more prominently in the articles on clinically applied medical anthropology. The relative prominence in usage of the term reflected a balance between the enthusiasm for the utility of explanatory models in clinical settings (Blumhagen, 1981), and modesty for claims of their anticipated contribution to ethnographic field research:

The explanatory models approach, however inadequate it may be for the ethnographer or novelist, brings meaning, person, family, feeling into the process of clinical judgement, and this opening to the humanness of suffering, in my experience, is often all that is needed to reaffirm for the physician the critical importance of psychological and social issues in a particular case and thereby make him less tolerant of delivering simply a technical 'fix'. (Kleinman, 1981, p. 375)

The journal Culture, Medicine, and Psychiatry (CMP) was first published in 1977, and this journal provided a forum that established the illness explanatory model framework in cross-cultural psychiatry, social medicine, and medical anthropology. Figure 10.1 presents an analysis of Medline citations for articles concerned with illness explanatory models from a textword search and manual review to exclude articles on non-illness explanatory models. Clinical and social medicine interests in the topic are reflected by a steady increase in Medline citations over the years. In the early 1980s, articles from $C M P$ constituted a major fraction, but now there are few. A full text search of the term in all CMP journal articles, however, through 2005 shows that attention to explanatory models as a term and concept firmly embedded in the literature remains relatively constant.

\section{Relevance for clinical practice and training}

Since the late 1970s, assertions of the value of explanatory models in clinical practice were based on two considerations. They provided a means of bridging cultural differences between patients and clinicians with different backgrounds in multicultural practice settings, and they also provided a means of bridging conceptual differences and promoting empathy and a therapeutic alliance, even when patients and clinicians came from similar cultural backgrounds. This was the argument that initially motivated development of an approach and efforts to promote clinically applied medical anthropology for general medical care (Kleinman, Eisenberg and Good, 1978),

Explanatory models were particularly important for both general psychiatry, where the same considerations for a clinical medical anthropology applied (Kleinman, 1978a), and for cultural psychiatry, which had additional compelling interests in questions of cultural validity, and which had become 


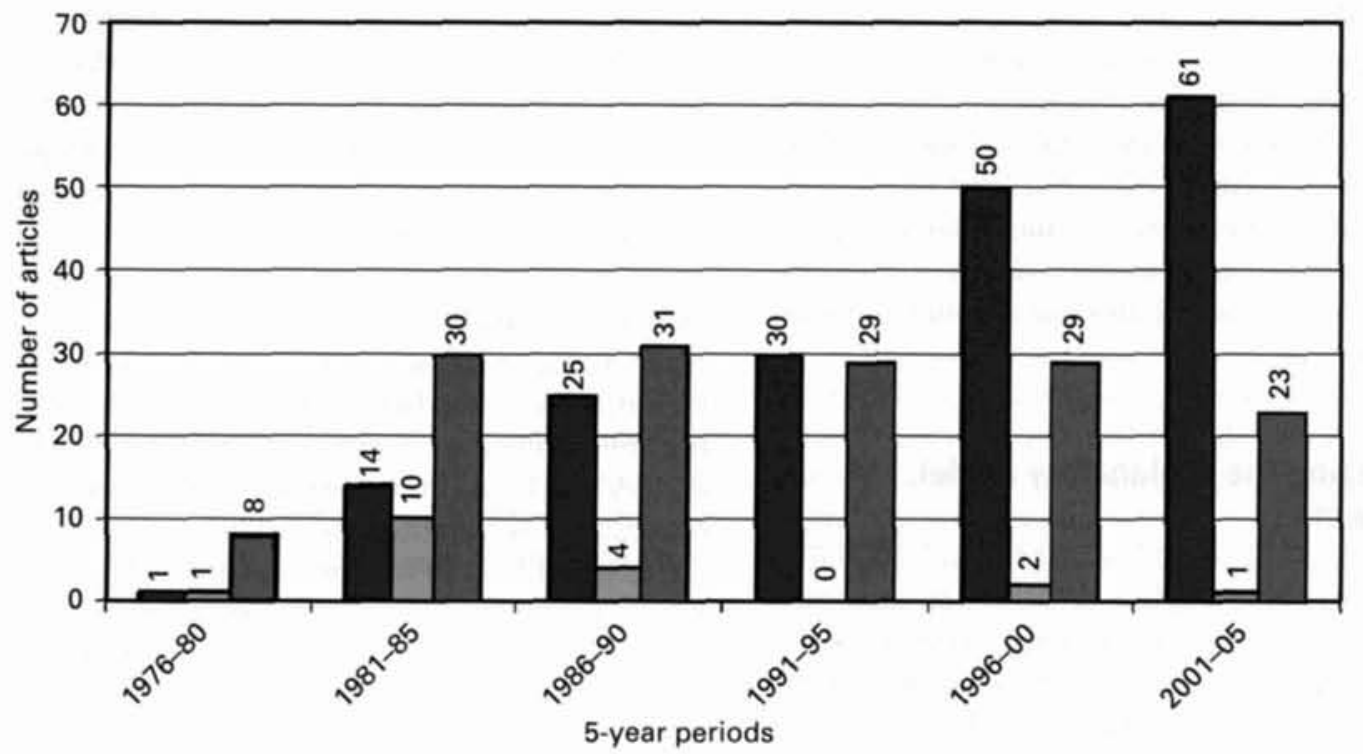

IIIness EMs in Medline $\square$ IIIness EMs Medline CMP only $\square$ IIIness EMs in CMP full text

Fig. 10.1. Medline articles on illness explanatory models and use of the term in the text of CMP articles.

sensitive to the potential for the misleading influence of category fallacies. Littlewood (1990) explained that a category fallacy resulted from mistaking professional Western explanatory models of mental illness for universal concepts of psychopathology. He also argued that an anthropologically informed approach to clinical practice served the interests of ethnic minorities; furthermore, attention to explanatory models elevated somewhat crass consideration of insight, which was typically reduced to the question of whether a patient agreed or disagreed with the doctor's views.

Advocacy for clinical attention to explanatory models also reflected concerns about an imbalance overemphasizing biology in the biopsychosocial model. Careful attention to patients' explanatory models, Kleinman had argued, may be regarded as a hallmark of empathy and ethical practice, and clinicians' failure to consider explanatory models of patients and their families could be taken as a mark of disrespect and arrogance (Kleinman, 1988a, p. 122). Such considerations suggested the need to ensure that supervision and training curricula recognize awareness and skill in working with explanatory models as a core clinical task, representing an important contribution of cultural psychiatry to clinical practice (Alarcón et al., 1999).

Recently, an editorial of Bhui and Bhugra (2002) renewed that call. Despite evidence that shared concepts of illness are associated with more satisfied patients (Callan and Littlewood, 1998), clinicians still lack the clinically relevant social science skills to assess and work with illness explanatory models skills that 'should be of prime importance in clinical psychiatric practice'. At the same time, interests in cultural competence in American medical education, which emphasize the value of working with explanatory models, suggest that such efforts to promote cultural sensitivity should be regarded as a mainstream, rather than marginal, feature of clinical training (Betancourt, 2004). A detailed curriculum with guidelines and examples has been developed 
for primary care by Carrillo and colleagues (1999). Exploring explanatory models, clarifying their context, and negotiating their implications with patients comprise the content of their module 3: Understanding the meaning of the illness.

The relevance of explanatory model for clinical assessment in psychiatry has been explicitly acknowledged in the outline of the cultural formulation in DSM-IV, Appendix I, and in the American Psychiatric Association's proposed research agenda for DSM-V (Mezzich et al., 1999; GAP, 2002; Alarcón, 2002). The cultural formulation was a product of the advisory group on culture and diagnosis, intended to provide a framework of clinical training that would enhance cultural sensitivity. It has been used for training in cultural psychiatry, and it has also been used as a guideline for case reports published in the psychiatric literature (LewisFernandez, 1996). The outline for the cultural formulation as a guideline for assessment requires elaboration of cultural explanations of the presenting illness. Although the interest of these cultural explanations is broad, the term explanatory model is used in the narrow sense, referring to the patient's 'perceived causes or explanatory models' among the broader features of the cultural explanation.

This section of the DSM-IV refers to another feature of cultural assessment with important historical implications, that of culture-bound syndromes. These also refer to a local configuration of illness, but they are associated with a conceptualization of an illness entity. The illness explanatory model, on the other hand, is concerned mainly with individual explanations of illness episodes, which may conform to a greater or lesser extent with various patterned illness entities - either professional disorders or local culture-bound syndromes - and the particular ways that both of these are related to an individual's experience and interpretation of illness.

\section{Critical assessment}

Countering the enthusiasm arising from the potential of explanatory models for bringing the influence of social science into medicine, there were also reservations. From the outset and thereafter, several critiques of the explanatory model framework have questioned its relevance. Although accounts of explanatory models repeatedly emphasize their fluidity, contradictions and shifting content, more akin to 'cognitive maps' (Kleinman, 1988a, p. 122), some authors find the framework too fixed and rigid. Williams and Healy (2001) characterize explanatory models as 'reified and implicitly static', suggesting an alternative formulation of 'exploratory map' to account for patients' definitions of their problems. A second critique is based on concerns that the clinical interests of explanatory models do not adequately account for the influence of social context. A third critique was based on the question of whether too much emphasis on the 'model' in explanatory model, which initially had been very appealing, was still a good idea.

A Marxist critique of medicine and the explanatory model framework developed in the late 1970s, first by Frankenberg (see Thomas, 1978). He argued that interests in the social determinants of health problems and failure to incorporate them adequately in the agenda of explanatory model studies rendered the enterprise inconsequential. Taussig, Frankenberg and Young argued that clinical paradigms were inherently inferior to political economic and social models of health and illness. Alan Young presented this argument in two papers, one in a provocative editorial published in CMP (Young, 1981) with eight rejoinders, and the second in an article on the anthropologies of illness and sickness in the Annual Review of Anthropology for 1982 (Young, 1982). He asserted that because Kleinman's interest in medical beliefs and practices is essentially clinical, it is inadequate to deal with more essential social priorities. He argued that analysis of the social relations of sickness identified two critical problems with the explanatory model approach; it confuses the class basis of power relationships with a feature of interpersonal relationships, and it fails to define sickness as 'a process for socializing disease and illness.'

Young advocated an alternative to illness explanatory models for studying 'socialized knowledge' of 
sickness, assessing prototypes and chain complexes. Stern and Kirmayer (2004) demonstrated that all three types of these illness representations - namely, explanatory models, prototypes, and chain complexes - could be assessed and reliably coded.

\section{The problem of models}

The appeal and the pitfalls of models for health systems and research were identified already in the first article of the first issue of CMP. Eisenberg (1977) pointed out that models were important and useful because they helped to construct reality and to lend meaning to a chaotic world. They are rightly regarded as particularly important for research because they determine the kind of questions we ask, the kind of data we gather, and the ways we analyse and interpret them. He cautioned, however, that 'models are indispensable but hazardous because they can be mistaken for reality itself rather than as but one way of organizing that reality' (p. 18).

Models were also identified with the important academic task of theory building. The use of the term explanatory model in the anthropological literature, before Kleinman introduced the illness explanatory model, referred to a formal theoretical description of a social phenomenon of interest for anthropological study. In that sense the term 'explanatory model' referred to an account that could successfully explain something. Nutini (1965), for example, reflecting on the task of model building wrote, 'Mechanical models constructed out of ideal behaviour, and statistical models based on actual behaviour are the best; they are the "most explanatory" models that we can build.' The various frameworks from and for medical anthropological research were all conceived as ethnomedical models, including products of Fabrega's work suggesting a multi-level schema for ethnomedicine, Eisenberg's disease-illness model, Good's semantic network model, and the explanatory model framework itself. Kleinman made that point in an editorial titled, 'Culture, and illness: a question of models' (Kleinman, 1977).
Before two decades had passed, however, their appeal as a guide to anthropological study had dwindled. It had not yet become clear how efforts to model the explanatory models might be turned into the kind of empirical research envisioned at the outset and then postponed. Kleinman himself appears to have accepted essential features of the Marxist critique of the clinical orientation and interests of explanatory models. Nearly two decades later, with academic interest by then focused more squarely on ethnography, he explained that he no longer respected the 'formalism, specificity, and authorial certainty' of any kind of models, including explanatory models. 'Clinically, the explanatory model approach may continue to be useful, but ethnography has fortunately moved well beyond this early formulation' (Kleinman, 1995, p. 9).

\section{Research on explanatory models}

The early promise of the explanatory model framework as a guide to ethnographic studies in medical anthropology and ethnomedicine may remain unfulfilled, but interest in the topic for clinical and cross-cultural research, especially in cultural psychiatry, is strong and growing. The value of acknowledging, comparing and accommodating different notions of illness in a globalizing world and in multicultural societies is difficult to disvalue or ignore. From a Medline search through the year 2005, which identified 181 articles concerned with illness explanatory models, 62 were concerned with psychiatry or mental health. Among single-disease studies, 10 focused exclusively on depression and 7 on schizophrenia; the remainder studied other conditions or a mix of disorders. About half of these articles report empirical data from clinic-based studies $(30,50.8 \%)$, and a smaller portion report community studies $(12,19.7 \%)$.

A review of this literature identifies several sets of interests, including applied clinical interests, fundamental questions in the field of cultural psychiatry and approaches to studying explanatory models. Brendel (2003) examined theoretical aspects of 
explanatory models, considering recent relevant work in the fields of ethics and philosophy of science. Other research suggests that consideration of clinician and patient explanatory models contributes to a more sophisticated explanation of insight for patients with schizophrenia (McGorry and McConville, 1999).

Among the clinically orientated publications, authors have been especially prolific on the topic of the cultural formulation. The journal CMP has a special section devoted to these case studies, and they are also published elsewhere, as much a feature of cultural psychiatry as case reports are in the general medical literature. Several studies indicate the utility of explanatory models in providing culturally sensitive care to immigrant patients (Daley, 2005; Bennegadi, 1996). Bhui and colleagues (2002) questioned whether the nature of patients' perceived causes of common mental disorders affected the likelihood of Punjabi and ethnic English patients in Britain receiving a diagnosis in primary care. Other clinical interests consider how to use information about illness explanatory models effectively in the course of psychotherapy. Anxiety attributed to a violent death in a previous life became the focus of therapy for a Druze patient in a case reported by Daie and colleagues (1992).

A focus of research that closely follows from historical interests in the field considers not only patients' explanatory models, but also those of clinicians and health workers. The negotiation of professional and patient ideas about problems like eating disorders, which are often contested conditions, becomes especially important (Swartz, 1987). Addressing different priorities, a study in South India noted that mental-health case workers had ideas about psychotic problems that diverged markedly from professional concepts (Joel et al., 2003). Sensitizing clinicians to the likely differences in concepts of mental-health problems between them and their patients has been recommended as a way to enhance the sensitivity of case finding among ethnic Chinese (Chan and Parker, 2004) and in Africa (Aidoo and Harpham, 2001). Based on experience in Goa and Harare, Patel (1995) suggests that emic research instruments are needed not only for culturally valid case identification (Rodrigues et al., 2003), but also for epidemiological studies. Research on depression in Bangalore, India, also considered the cultural validity of depression, anxiety and somatoform disorders by examining the relationship between emic concepts and professional diagnoses (Weiss et al., 1995).

Practical questions of behaviour concerned with the influence of explanatory models on patterns of help seeking have remained matters of interest for planning community mental-health services. Research has examined the influence of such ideas about illness on help seeking in child psychiatry clinics in Hong Kong (Ho and Luk, 1997), for panic disorders in Lesotho (Hollifield et al., 1990), and among various ethnic groups in Britain (Sheikh and Furnham, 2000). Several studies also indicate the value of examining the effects of illness explanatory models on adherence to treatment for various psychiatric and medical conditions (Weiss et al., 1992; Wong et al., 1999).

Explanatory-model studies have been applied to elaborate the cultural context and meaning of various mental disorders, including schizophrenia (Larsen, 2004; Niehaus et al., 2004), depression among elderly Koreans (Pang, 1998), other psychiatric conditions, and acquisition of a non-specific identity as a psychiatric patient (Sayre, 2000). With reference to cultural and historical context, studies have also considered how current experience and meaning of psychiatric disorders in India relates to classical humoral concepts and medical traditions of Ayurveda (Weiss et al., 1986). Lee's (1995) research on anorexia suggests that efforts to associate that condition with Western explanatory models may result in a category fallacy. He argues that the disorder is fundamentally determined by cultural influences and may lack a core psychopathology.

Research has also used the framework of explanatory models for various cross-cultural comparisons. These include ideas about mental distress (Eisenbruch, 1990), schizophrenia in four cultural groups (McCabe and Priebe, 2004), and ideas about 
substance misuse among German and Turkish youth in Germany (Penka et al., 2003). Focused studies on determinants of undesirable outcomes have considered the role of explanatory models in a study of use of traditional healers for obsessive-compulsive disorder and Tourette's syndrome in Bali (Lemelson, 2004). Ethnographic data from interviews with participants of an internet community with a 'proanorexia' anti-treatment agenda consider their underlying explanatory models, showing how they diverge from the views of health professionals and others who regard anorexia nervosa as a dangerous disorder (Fox, Ward and O'Rourke, 2005).

\section{Studying explanatory models and directions for research}

Despite inconsistencies in the way that it is understood, the diverse interests of explanatory model research briefly reviewed above suggest that illness explanatory models are likely to remain an important interest of cultural psychiatry. These studies have developed in various ways, and with reference to broad, narrow and intermediate formulations of the concept of illness explanatory model. They have also used various research methods. Some studies elicit explanatory models with open-ended questions in the style of a clinical interview. Some investigators refer to the eight questions Kleinman suggested in Patients and Healers (1980, p. 106n) as a guide, or include some modification (Aidoo and Harpham, 2001). More open-ended assessment may also rely on motivating illness narratives from which qualitative analysis extracts explanatory models. The illness narrative technique is especially useful for elaborating detail and context (Kleinman, 1988a), but may present problems for working with variables suitable for specific comparisons and testing hypotheses. In practice, to specify the explanatory models or other conceptualizations derived from the narrative, an approach to coding is required (Stern and Kirmayer, 2004).

Efforts to construct a brief, semi-structured interview to elicit illness explanatory models efficiently led to the development of the Short Explanatory Model Interview (SEMI) by Lloyd and colleagues (1998). Constructed in the style of a psychiatric epidemiological assessment, it inquired about the essential features of the illness explanatory model of patients in primary care, consistent with the accounts of Kleinman, interests of illness narratives and $\mathrm{a}$ formulation embodied in earlier explanatorymodel interviews (Weiss 1997, 2001). The SEMI was field tested with a sample of three ethnic groups in London and in Harare. In both studies, patients with suspected common mental disorders were asked about their presenting problems, and in the London sample patients were also asked to comment on two vignettes depicting symptoms of depression and somatization. Subsequent research has also used the SEMI to study explanatory models of psychosis among mental-health workers (Joel et al., 2003) and patients representing four ethnic groups (McCabe and Priebe, 2004).

Earlier explanatory model interviews, known collectively as Explanatory Model Interview Catalogue (EMIC interviews) (Weiss 1997, 2001), were initially developed in Mumbai and Bangalore with separate versions for study of patients with leprosy (Weiss et al., 1992) and psychiatric disorders in outpatient clinics (Weiss et al., 1995). Conceived as an approach for systematically studying a broad formulation of explanatory models for different health problems in different settings (clinics and communities), there is no single definitive EMIC interview, but rather a family of instruments, each constructed with reference to a common framework, and an adaptation constructed to accommodate questions motivating a particular research study. The term EMIC was initially conceived both to designate the local, i.e. emic, perspective and as an acronym for explanatory-model interview catalogue. These interviews remain interested in the local emic account of illness. The distribution of categories, informed by complementary qualitative data, constitutes a cultural epidemiology of representations of illness that collectively may be regarded as an emic account or an explanatory model suitable for empirical study. 
Each EMIC interview is associated with a particular study and locally adapted. Their structure typically includes sections concerned with illnessrelated experience, meaning, and behaviour, formulated in open-ended and category-specific probing questions that inquire about patterns of distress, perceived causes, and help-seeking and risk-related behaviour. The coding is designed to facilitate comparisons, and analysis of the relationship between features of illness explanatory models and outcomes of practical clinical interest or public-health significance. The data sets typically include category codes and illness narratives linked to questions of the interview, so that the structure may facilitate integrated analysis of quantitative and qualitative components of a data set, aided by use of appropriate software.

\section{Conclusions}

The concept of illness explanatory models developed by Arthur Kleinman arose during a fertile period in the development of clinically applied medical anthropology, and it remains a useful approach for bridging the interests and experience of clinicians and their patients. Although broadly conceived, especially at the outset, as a framework for advancing cultural psychiatry, enhancing cultural sensitivity and psychosocial interests of clinical practice, and contributing to the development of ethnomedical studies, it has become less appealing as a framework for ethnography because the clinical orientation of explanatory models did not fulfil initial promise as a guide for social analysis. Subsequent advances in medical anthropology have not been particularly concerned with backward compatibility in that regard. Nevertheless, the concept of explanatory models has been firmly established in the lexicon of culture, health and illness studies and remains highly valued for eliciting a perspectivist account of illness. Attention to explanatory models remains as much a priority as ever, because working with them enhances empathy, respect and a therapeutic alliance.
Although attention to explanatory models remains well justified for inclusion in psychiatric and medical curricula, questions about the significance and implications of findings from explanatory model studies remain. How well do explanatory models predict behaviour? What particular features of explanatory models are most important in that regard? How do explanatory models relate to other sociocultural features of health and health problems, such as stigma and gender? In addition to enduring clinical interests, these are the questions that should motivate further study of explanatory models.

Concern about promoting technological fixes and an exclusively biological approach to essential features of health policy and clinical practice that are essentially social and cultural remains salient. Health social-science research, especially cultural epidemiology, and culturally sensitive clinical practice and training continue to benefit from a formulation of illness explanatory models that suits their use, and more so when their study is complemented by attention to political, economic and social forces that influence health and illness.

\section{References}

Aidoo, M. and Harpham, T. (2001). The explanatory models of mental health amongst low-income women and health care practitioners in Lusaka, Zambia. Health Policy and Planning. 16, 206-213.

Alarcón, R. D., Alegria, M., Beil, C. C. et al, (2002). Beyond the funhouse mirrors: research agenda on culture and psychiatric diagnosis. In: $A$ Research Agenda for $D S M-V$, ed. D.G. Kupfer, M. B. First, and D.A. Regier. Washington, DC: American Psychiatric Association.

Alarcón, R.D., Westermeyer, J., Foulks, E. F. and Ruiz, P. (1999). Clinical relevance of contemporary cultural psychology. Journal of Nervous and Mental Disease, 187, 465-471.

Bennegadi, R. (1996). Clinical medical anthropology and immigrant's mental health in France. Médecine Tropicale, 56, 445-452. 
Betancourt, J. R. (2004). Cultural competence - marginal or mainstream movement? New England Journal of Medicine, 351, 953-955.

Bhui, K. and Bhugra, D. (2002). Explanatory models for mental distress: implications for clinical practice and research. British Journal of Psychiatry, 181, 6-7.

Bhui, D., Bhugra, D. and Goldberg, D. (2002). Causal explanations of distress and general practitioners' assessments of common mental disorder among Punjabi and English attendees. Social Psychiatry and Psychiatric Epidemiology, 37, 38-45.

Blumhagen, D.W. (1981), On the nature of explanatory models. Culture, Medicine and Psychiatry, 5, 337-340.

Brendel, D.H. (2003). Reductionism, eclecticism and pragmatism in psychiatry: the dialectic of clinical explanation. Journal of Medicine and Philosophy, 28, $563-580$.

Callan, A. and Littlewood R. (1998). Patient satisfaction: ethnic origin or explanatory model? International Journal of Social Psychiatry, 44, 1-11.

Carrillo, J. R., Green, A. R. and Betancourt, J. R. (1999). Cross-cultural primary care: a patient-based approach. Annals of Internal Medicine, 130, 829-834.

Chan, B. and Parker, G. (2004). Some recommendations to assess depression in Chinese people in Australasia. Australian and New Zealand Journal of Psychiatry, 38, 141-147.

Daie, N., Witztum, E., Mark, M. and Rabinowitz, S. (1992). The belief in the transmigration of souls: psychotherapy of a Druze patient with severe anxiety reaction. British Journal of Medical Psychology, 65, 119-130.

Daley, T.C. (2005). Beliefs about treatment of mental health problems among Cambodian American children and parents. Social Science and Medicine, 61, 2384-2395.

Eisenberg, L. (1977). Disease and illness: distinctions between professional and popular ideas of sickness. Culture, Medicine and Psychiatry, 1, 9-23.

Eisenbruch, M. (1990). Classification of natural and supernatural causes of mental distress: development of a Mental Distress Explanatory Model Questionnaire. Journal of Nervous and Mental Disease, 178, 712-719.

Engel, G. L. (1977). The need for a new medical model: a challenge for biomedicine. Science, 196, 129-136

Fabrega, H., Jr. (1975). The need for an ethnomedical science. Science, 189, 969-975.

Fabrega, H., Jr. (1990). A plea for a broader ethnomedicine. Culture, Medicine and Psychiatry, 14, 129-132.

Fox, N., Ward, K. and O'Rourke, A. (2005). Pro-anorexia, weight-loss drugs and the internet: an 'anti-recovery' explanatory model of anorexia. Sociology of Health and Illness, 27, 944-971.

Good, B. (1977). The heart of what's the matter: the semantics of illness in Iran. Culture, Medicine and Psychiatry, 1, 25-58.

Group for the Advancement of Psychiatry (2002). Cultural Assessment in Clinical Psychiatry. GAP Publication, \#145. Committee on Cultural Psychiatry. Washington, DC: American Psychiatric Press.

Headland, T. N., Pike, K. L. and Harris M., eds. (1990). Emics and Etics: The Insider/Outsider Debate. Newbury Park, CA: Sage.

Helman, C. (2004). Culture, Health, and Illness, 4th edn. London: Hodder Amold.

Ho, T. P. and Luk, C. (1997). Comparison of child psychiatric patients in hospital and community clinics in Hong Kong. General Hospital Psychiatry, 19, 362-369.

Hollifield, M., Katon, W., Spain, D. and Pule, L. (1990), Anxiety and depression in a village in Lesotho, Africa: a comparison with the United States. British Journal of Psychiatry, 156, 343-350.

Joel, D., Sathyaseelan, M., Jayakaran, R., Vijayakumar, C., Muthurathnam, S. and Jacob, K.S. (2003). Explanatory models of psychosis among community health workers in South India. Acta Psychiatrica Scandinavica, 108, 66-69.

Kleinman, A. (1977a). Culture, and illness: a question of models. Culture, Medicine and Psychiatry, 1, 229-231.

Kleinman, A. (1977b). Depression, somatization and the 'new cross-cultural psychiatry'. Social Science and Medicine, 11, 3-10.

Kleinman, A. (1978a). Clinical relevance of anthropological and cross-cultural research: concepts and strategies. American Journal of Psychiatry. 135, 427-431.

Kleinman, A. (1978b). What kind of model for the anthropology of medical systems? American Anthropologist, $\mathbf{8 0}$, 661-665.

Kleinman, A. (1980). Patients and Healers in the Context of Culture: An Exploration of the Borderland between Anthropology, Medicine, and Psychiatry. Berkeley: University of California Press.

Kleinman, A. (1981), On illness meanings and clinical interpretation: not 'rational man,' but a rational approach to man the sufferer/man the healer. Culture, Medicine, and Psychiatry, 4, 373-377.

Kleinman, A. (1986). Social Origins of Distress and Disease: Depression, Neurasthenia and Pain in Modern China. New Haven: Yale University Press, p. 84.

Kleinman, A. (1988a). The Illness Narratives: Suffering, Healing and the Human Condition. New York: Basic Books. 
Kleinman, A. (1988b). Rethinking Psychiatry: From Cultural Category to Personal Experience. London: Free Press.

Kleinman, A. (1995). Writing at the Margin of Discourse between Anthropology and Medicine. Berkeley: University of California Press.

Kleinman, A., Eisenberg, L. and Good, B. (1978). Culture, illness and care: clinical lessons from anthropologic and cross-cultural research. Annals of Internal Medicine, 88, 251-258.

Larsen, J. A. (2004). Finding meaning in first episode psychosis: experience, agency and the cultural repertoire. Medical Anthropology Quarterly, 18, 447-471.

Lee, S. (1995). Self-starvation in context: towards a culturally sensitive understanding of anorexia nervosa. Social Science and Medicine, 41, 25-36.

Lemelson, R. B. (2004). Traditional healing and its discontents: efficacy and traditional therapies of neuropsychiatric disorders in Bali. Medical Anthropology Quarterly, 18, 48-76.

Lewis-Fernandez, R. (1996). Cultural formulation of psychiatric diagnosis. Culture, Medicine and Psychiatry, 20, 133-144.

Littlewood, R. (1990). The new cross-cultural psychiatry. British Journal of Psychiatry, 157, 775-776.

Lloyd, K. R., Jacob, K. S., Patel, V., St Louis L., Bhugra, D. and Mann, A. H. (1998). The development of the Short Explanatory Model Interview (SEMI) and its use among primary-care attenders with common mental disorders. Psychology and Medicine, 28, 1231-1237.

McCabe, R. and Priebe S. (2004). Explanatory models of illness in schizophrenia; comparison of four ethnic groups. British Journal of Psychiatry, 185, 25-30.

McGorry, P. D. and McConville, S. B. (1999). Insight in psychosis: an elusive target. Comprehensive Psychiatry, 40, 131-142.

Mezzich, J. E., Kirmayer, L. J., Kleinman, A. et al. (1999). The place of culture in DSM-IV. Journal of Nervous and Mental Disease, 187, 457-464.

Niehaus, D. J., Oosthuizen, P., Lochner, C. et al. (2004). A culture-bound syndrome amafufunyana and a culturespecific event ukuthwasa: differentiated by a family history of schizophrenia and other psychiatric disorders. Psychopathology, 37, 59-63.

Nutini, H.G. (1965) Some considerations on the nature of social structure and model building: a critique of Claude Levi-Strauss and Edmund Leach. American Anthropologist, 67, 707-731.
Pang, K.Y. (1998). Symptoms of depression in elderly Korean immigrants: narration and the healing process. Culture, Medicine and Psychiatry, 22, 93-122.

Patel, V. (1995). Explanatory models of mental illness in sub-Saharan Africa. Social Science and Medicine, 40, 1291-1298.

Penka, S., Krieg, S., Hunner, Ch. and Heinz, A. (2003). Different explanatory models ofr addictive behavior in Turkish and German youths in Germany: significance for prevention and treatment. Nervenarzt, 74, 581-586.

Pike, K. L. (1967). Etic and emic standpoints for the description of behavior. In: ed. Language and Thought: An Enduring Problem in Psychology, D.C., Hildum, Princeton: Van Nostrand.

Rodrigues, M., Patel, V., Jaswal, S. and de Souza, N. (2003). Listening to mothers: qualitative studies on motherhood and depression from Goa, India. Social Science and Medicine, 57, 1797-1806.

Sayre, J. (2000). The patient's diagnosis: explanatory models of mental illness. Qualitative Health Research, 10, 71-83.

Sheikh, S. and Furnham, A. (2000). A cross-cultural study of mental health beliefs and attitudes towards seeking professional help. Social Psychiatry and Psychiatric Epidemiology, 35, 326-334.

Stern, L. and Kirmayer, L. J. (2004). Knowledge structures in illness narratives: development and reliability of a coding scheme. Transcultural Psychiatry, 41, 130-142.

Swartz, L. (1987). Illness negotiation: the case of eating disorders. Social Science and Medicine, 24, 613-618.

Thomas, A. (1978). Discussion on Arthur Kleinman's paper. Social Science and Medicine, 12, 95.

Weiss, M.G. (1997). Explanatory Model Interview Catalogue: framework for comparative study of illness experience. Transcultural Psychiatry, 34, 235-263.

Weiss, M.G. (2001). Cultural epidemiology: an introduction and overview. Anthropology and Medicine, 8, $5-29$.

Weiss, M. G., Sharma, S. D., Gaur, R. K., Sharma, J. S., Desai, A. and Doongaji, D. R. (1986). Traditional concepts of mental disorder among Indian psychiatric patients: preliminary report of work in progress. Social Science and Medicine, 23, 379-386.

Weiss, M. G., Doongaji, D. R., Siddhartha, S. et al. (1992). The Explanatory Model Interview Catalogue (EMIC). Contribution to cross-cultural research methods from a study of leprosy and mental health. British Journal of Psychiatry, 160, 819-830. 
Weiss, M. G., Raguram, R. and Chamnabasavanna, S.M. (1995). Cultural dimensions of psychiatric diagnosis. A comparison of DSM-III-R and illness explanatory models in South India. British Journal of Psychiatry, 166, 353-359.

Williams, B. and Healy, D. (2001). Perceptions of illness causation among new referrals to a community mental health team: explanatory model or exploratory map? Social Science and Medicine, 53, 465-476.
Wong, S. S., Lee, S. and Wat, K.H. (1999). A preliminary communication of an insight scale in the assessment of lithium non-adherence among Chinese patients in Hong Kong. Journal of Affective Disorders, 44, 241-244. Young, A. (1981). When rational men fall sick: an inquiry into some assumptions made by medical anthropologists. Culture, Medicine and Psychiatry, 5, 317-337.

Young, A. (1982). The anthropologies of illness and sickness. Annual Review of Anthropology, 11, 257-285. 